\title{
Mechanical Etiologies Associated with the Diagnosis of Esophageal Outflow Obstruction on High Resolution Manometry
}

\author{
Brian E Jones and Steven B Clayton* \\ Assistant Professor of Internal Medicine, Wake Forest University Baptist Medical Center, USA
}

Submission: October 01, 2020; Published: October 09, 2020

*Corresponding author: Steven B Clayton, Assistant Professor of Internal Medicine, Wake Forest University Baptist Medical Center, Medical Center Blvd. Winston-Salem, NC 27157, USA

\section{Abstract}

Background and Aims: Esophageal anatomic obstruction has been associated with the manometric diagnosis of esophagogastric junction outflow obstruction (EGJ00). Endoscopic evaluation is usually the initial step to evaluate dysphagia in adults; however, many high-resolution impedance manometries (HRIM) are being ordered prior to endoscopy especially in open access settings. The aim of this study was to analyze and describe anatomic findings associated with the manometric diagnosis of EGJOO.

Materials and Methods: A retrospective chart review was performed on all patients receiving HRIM. Clinical presentation, diagnostic testing, and treatment findings were recorded. Patients were categorized as having anatomic versus idiopathic etiologies using all available records. The subgroups were described and evaluated.

Results: 72 of 114 patients were identified as having anatomic EGJOO. The most common anatomic findings associated EGJOO were hiatal hernia (59.7\%), esophagitis (31.9\%), strictures/webs/Schatzki's rings $(26.4 \%)$ and fundoplication (26.4\%). $21.9 \%$ of patients with HRIM findings consistent with EGJOO had history of prior relevant surgery. Most patients with an identified anatomic etiology had symptom response to directed treatment.

Conclusions: Anatomic findings, such as hiatal hernias and post-surgical changes, are commonly associated with EGJOO on HRIM. Postsurgical HRIM findings consistent with EGJOO, likely secondary to anatomic changes (e.g. following fundoplication), represent a higher portion of our studied population than previously described in the literature. Other than EGD diagnostic test are not able to significantly distinguish between anatomic and idiopathic EGJ00. EGD should continue to be performed prior to HRIM and if not already performed, on any patient with the diagnosis of EGJOO.

Keywords: Endoscopy; Digestive System; Esophageal Diseases; Dilation; Hernia, Hiatal; Manometry

\section{Introduction}

Considerable clinical ambiguity exists over the relevance, optimal evaluation, and treatment of the manometric diagnosis of esophagogastric junction outflow obstruction (EGJO0). Additional complexity arises as the manometric diagnosis of EGJOO, can be caused by either anatomic (also known as mechanical in the medical literature) esophageal etiologies or a poorly relaxing lower esophageal sphincter (LES) [1-8]. Ideally, endoscopic evaluation is performed to evaluate dysphagia in adults prior to high-resolution impedance manometry (HRIM); but many times HRIM is completed prior to endoscopic evaluation. This may be a result of centers allowing open access ordering of HRIM by non-gastroenterology providers. For example, current Society of American Gastrointestinal and Endoscopic Surgeons (SAGES) guidelines acknowledge both EGD and HRIM as preoperative evaluations among other test and admit that there is significant variability among surgeons on which test are performed and the order they are obtained [9].

Anatomic EGJOO has been associated with the following: esophageal strictures, hiatal hernia, prior fundoplication, adjustable gastric band surgery, eosinophilic esophagitis, and malignancy [1]. The idiopathic form of EGJOO is defined as an elevated median integrated relaxation pressure (IRP) ( $\geq 15 \mathrm{mmHg}$ ) with preserved peristalsis on HRIM, such that the criteria of achalasia are not met but no anatomic esophageal obstruction exists.(7, 8, 10) Many studies have focused on the idiopathic form of EGJOO but few have performed an in-depth examination of the 
esophageal anatomic findings associated with the manometric diagnosis.

The aim of this study was to analyze and describe anatomic findings associated with the manometric diagnosis of EGJOO. Secondary aims were to evaluate clinical presentation, HRIM, liquid impedance analysis and other radiologic findings on standard barium esophagram (SBE) and timed barium esophagram (TBE) that distinguish between anatomic versus idiopathic EGJOO.

\section{Materials and Methods}

All high-resolution impedance manometries, including open access studies, performed over a two-year period at large tertiary academic system were analyzed. Inclusion criteria were adult patients ( $\geq 18$ years old) with EGJOO as defined by the Chicago Classification v3.0 (IRP median over 10 swallows $\geq 15.0 \mathrm{mmHg}$ with intact peristalsis) [10]. An extensive chart review recorded patient demographics, clinical presentation, HRIM parameters, incomplete bolus clearance on impedance analysis, TBE results, SBE findings, esophagogastroduodenoscopy (EGD) findings, relevant surgical history, history of gastroesophageal reflux disease (GERD), and opioid use. Review of protected medical information was approved without the need for waiver of consent given low risk nature of the study by the institutional review board of Greenville Health System (now Prisma Health). Treatment methodologies, treatment sequence, and patient's subjective response to treatment were recorded. A patient's response to treatment was defined as a subjective improvement in presenting symptoms documented in the medical record.

\section{Timed Barium Esophagram Protocol}

TBE was obtained using a standardized protocol. The patient was administered $240 \mathrm{ml}$ (8oz.) of low-density barium in the standing position; spot films were obtained at 1 minute and 5 minutes to assess liquid emptying. Barium column height as measured from the gastroesophageal (GE) junction to the top of the column was recorded. Next, the esophagus was cleared with water followed by ingestion of a 13-mm barium tablet. Tablet passage was evaluated after 5 minutes with abnormal test being tablet retention. The following parameters were recorded: barium height at 1 and 5 minutes in centimeters, barium width at 1 and 5 minutes in centimeters, and tablet retention.

\section{High Resolution Manometry Protocol}

The esophageal motor function of all patients was studied with a 36-channel solid-state catheter system with high fidelity circumferential sensors at $1 \mathrm{~cm}$ intervals and intra-luminal impedance (Medtronic, Inc., Minneapolis, MN, USA). The manometry catheter was placed transnasally and positioned to record from the hypopharynx to the stomach with approximately five intragastric sensors. Studies were performed in the supine position after at least 6 hours of fasting. The protocol included a 3-minute baseline period and at least ten 5-ml water swallows, each separated by 30 -second intervals. All data were analyzed using ManoView software in the high-resolution esophageal color topography mode to standardize data analysis. All pertinent metric data were recorded, including lower esophageal sphincter basal pressure, intrabolus pressure (IBP), IRP, distal contractile integral (DCI), large breaks, contractile frontal velocity, and distal latency (DL) [10-12]. The Chicago Classification version 3.0 criteria were used to establish the diagnosis of EGJOO and classify esophageal dysmotility [10].

\section{Identification of Anatomic Versus Idiopathic Etiology}

All available records were reviewed to identify anatomic esophageal obstruction versus an idiopathic etiology. The EGD findings were reviewed by a gastroenterologist with experience in esophageal disease who assigned each record to anatomic and idiopathic subcategories based on EGD findings. Records with EGD findings including the following, except for in cases where a rosette was also identified, were placed in the anatomic subgroup: gastroesophageal reflux disease with erosive esophagitis, stricture/web/ring, esophageal diverticulum, esophageal ulcer, eosinophilic esophagitis, fundoplication, varices, esophageal nodules, and hiatal hernia. All other patients were identified as having idiopathic EGJOO.

In addition to endoscopy, hiatal hernias were identified via barium esophagram or HRIM. Hiatal hernia size was defined as the distance from the gastric folds to the diaphragmatic hiatus/ impression on endoscopy, the distance of abdominal contents through the esophageal hiatus of the diaphragm into the thoracic cavity on esophagram, and/or the distance between the LES pressure zone and the crural diaphragm pressure zone on HRIMs where a double pressure zone distally on the Clouse plot was present.

\section{Statistical Methods}

Descriptive statistics (means and standard deviations for continuous factors, frequencies and percentages for categorical factors) were computed by etiology type (anatomic versus idiopathic). For continuous factors, statistical tests for differences in means between the groups were assessed using two-group t-tests. For categorical factors, differences in proportions between groups were assessed using chi-square tests or Fisher's exact test in cases of $2 \times 2$ tables where expected cell counts were small (i.e., $<5)$. Instances of low expected cell counts when Fisher's exact test could not be used because the table was not square are noted in the results. No adjustment of p-values for propagation of type I error due to multiple hypothesis testing was performed, thus statistical significance (i.e., p-values $<0.05$ ) should be interpreted with that caveat in mind. All analyses were performed using SAS statistical software, version 9.4 (SAS Institute, Cary, NC, USA) 
Results

A total of 114 patients met inclusion criteria. Clinical presentation of these patients is described in Table 1. No significant difference between the anatomic and idiopathic subgroups in terms of demographics, clinical presentation, chief complaint (indication for HRIM), presence or type of GERD, presence or type of dysphagia or history of opioid use was found. Prior history of upper GI surgery (fundoplication, hiatal hernia repair with/ without fundoplication, neck/chest radiation) was seen more commonly in patients of the anatomic subgroup $(\mathrm{p}=0.001)$.

Table 1: Demographics and Chief Complaints of Anatomic vs. Idiopathic Subtypes.

\begin{tabular}{|c|c|c|c|}
\hline & Anatomic & Idiopathic & p-value \\
\hline $\mathrm{N}$ & 72 & 42 & \\
\hline Age, Mean \pm SD & $59.4 \pm 12.0$ & $55.6 \pm 13.4$ & 0.12 \\
\hline \multicolumn{4}{|l|}{ Sex, N (\%) } \\
\hline Male & $30(41.7)$ & $18(42.9)$ & \multirow{2}{*}{0.9} \\
\hline Female & $42(58.3)$ & $24(57.1)$ & \\
\hline \multicolumn{4}{|l|}{ Chief Complaint } \\
\hline Dysphagia & $30(41.7)$ & $23(54.8)$ & \multirow{7}{*}{$0.56^{*}$} \\
\hline Burning/Regurgitation/Reflux & $17(23.6)$ & $9(21.4)$ & \\
\hline Extra-esophageal manifestation of GERD $†$ & $13(18.1)$ & $3(7.1)$ & \\
\hline Chest Pain & $7(9.7)$ & $4(9.5)$ & \\
\hline Abdominal Pain/Epigastric Pain & $2(2.8)$ & $2(4.8)$ & \\
\hline Choking & $2(2.8)$ & 0 & \\
\hline Esophageal Spasms & $1(1.4)$ & $1(2.4)$ & \\
\hline Prior Related Surgery $\ddagger$ & $25(34.7)$ & 0 & $<0.001$ \\
\hline Fundoplication, N (\% prior surgery) & $21(84.0)$ & 0 & \\
\hline Hiatal Hernia & $7(28.0)$ & 0 & \\
\hline Other Surgeries & $4(16)$ & 0 & \\
\hline Dysphagia & $55(76.4)$ & $34(81.0)$ & 0.57 \\
\hline None & $17(23.6)$ & $8(19.0)$ & \multirow{5}{*}{$0.51^{*}$} \\
\hline Solid/Pill N (\% dysphagia) & $29(52.7)$ & $20(58.8)$ & \\
\hline Liquid & $1(1.8)$ & $2(5.9)$ & \\
\hline Both & $19(34.5)$ & $11(32.4)$ & \\
\hline Unknown/Undocumented & $6(10.9)$ & $1(2.9)$ & \\
\hline GERD & $56(77.8)$ & $34(81.0)$ & 0.69 \\
\hline None & $16(22.2)$ & $8(19.0)$ & \multirow{5}{*}{$0.51^{*}$} \\
\hline Typical (Heartburn, Regurgitation) (\% GERD) & $39(69.6)$ & $28(82.4)$ & \\
\hline Atypical & $6(10.7)$ & $3(8.8)$ & \\
\hline Both & $10(17.9)$ & $3(8.8)$ & \\
\hline Asymptomatic/Noted on Studies & $1(1.8)$ & 0 & \\
\hline Opioid Use at Time of HRIM $\S$ & $33(45.8)$ & $12(28.6)$ & 0.069 \\
\hline \multicolumn{4}{|c|}{ *At least one expected cell count $<5$, chi-square test should be interpreted with caution } \\
\hline \multicolumn{4}{|c|}{$\begin{array}{c}\text { †Gastrointestinal reflux disease } \\
\text { ‡Some patients with prior related surgeries had history of multiple procedures } \\
\S \text { High resolution impedance manometry }\end{array}$} \\
\hline
\end{tabular}

Diagnostic study results including: HRIM metrics, liquid bolus transit on impedance analysis, TBE, and SBE showed no significant differences between the subgroups. A summary of radiographic evaluation with TBE and SBE can be seen in Table 2. 


\section{Advanced Research in Gastroenterology \& Hepatology}

Table 2: Radiographic Study Findings in Anatomic vs. Idiopathic Subtypes.

\begin{tabular}{|c|c|c|c|}
\hline & Anatomic & Idiopathic & p-value \\
\hline $\mathrm{N}$ & 72 & 42 & \\
\hline Hernia per HRIM $\dagger$ & $18(25.0)$ & $8(19.0)$ & 0.47 \\
\hline Size, Mean \pm SD & $2.7 \pm 1.6$ & $2.0 \pm 1.0$ & 0.25 \\
\hline Timed Barium Esophagram & $40(55.6)$ & $21(50.0)$ & 0.57 \\
\hline Normal, N (\% TBE $\ddagger)$ & $17(42.5)$ & $10(47.6)$ & 0.7 \\
\hline $1^{\prime}(\mathrm{N}=27)$, Mean $\pm \mathrm{SD}$ & (18) $11.3 \pm 5.1$ & (9) $11.9 \pm 6.2$ & 0.77 \\
\hline $5^{\prime}(\mathrm{N}=11)$, Mean $\pm \mathrm{SD}$ & (7) $10.8 \pm 5.8$ & (4) $4.9 \pm 3.7$ & 0.11 \\
\hline Tablet Passed (N=44), N (\%) & $26(65.0)$ & $18(85.7)$ & 0.086 \\
\hline Standard Barium Esophagram, N (\%) & $50(69.4)$ & $26(61.9)$ & 0.41 \\
\hline Dysmotility (\%SBEף§) & $22(44.0)$ & $10(38.5)$ & 0.64 \\
\hline Hiatal Hernia & $20(40.0)$ & $5(19.2)$ & 0.068 \\
\hline Narrow Gastroesophageal Junction & $8(16.0)$ & $8(30.8)$ & 0.13 \\
\hline Reflux & $11(22.0)$ & $2(7.7)$ & $0.20^{*}$ \\
\hline Arrested/Delayed Tablet & $9(18.0)$ & $2(7.7)$ & $0.31 *$ \\
\hline Esophageal Stricture/Schatzki's Ring & $9(18.0)$ & $1(3.8)$ & $0.15^{*}$ \\
\hline Fundoplication & $7(14.0)$ & $0(0.0)$ & $0.088^{*}$ \\
\hline Barium Aspiration & $4(8.0)$ & $2(7.7)$ & $1.00^{*}$ \\
\hline Diverticula & $4(8.0)$ & $1(3.8)$ & $0.66^{*}$ \\
\hline Enlarged Cricopharyngeal Muscle/ & $4(8.0)$ & $0(0.0)$ & $0.29 *$ \\
\hline Prolonged Barium Retention & $3(6.0)$ & $3(11.5)$ & $0.41^{*}$ \\
\hline Dilation & $2(4.0)$ & $1(3.8)$ & $1.00^{*}$ \\
\hline
\end{tabular}

*Due to low expected cell count, p-value from Fisher's exact test for square 2 -way tables

†High resolution impedance manometry

$\ddagger$ Timed Barium Esophagram

§Many patients had several different findings on SBE

ףTStandard Barium Esophagram

Using EGD n=72, (63.2\%) anatomic cases and $n=42,(36.8 \%)$ idiopathic cases were identified. EGD findings of those records assigned an anatomic etiology are seen in Table 3. Table 4

describes further information on anatomic causes identified in patients with and without history of opioid use.

Table 3: Esophagogastroduodenoscopy Findings of Patients with Anatomic Etiology.

\begin{tabular}{|c|c|}
\hline Patients with Anatomic Causes, $\mathbf{~}$ & $\mathbf{7 2}$ \\
\hline Hiatal Hernia, N (\%) $\dagger$ & $43(59.7)$ \\
\hline Gastritis & $36(50)$ \\
\hline Erosive Esophagitis $\dagger$ & $23(31.9)$ \\
\hline Stricture/Schatzki's ring/Web $\dagger$ & $19(26.4)$ \\
\hline Fundoplication $\dagger$ & $19(26.4)$ \\
\hline Dysmotility & $13(18.1)$ \\
\hline Barrett's Esophagus & $11(15.3)$ \\
\hline Duodenitis & $8(11.1)$ \\
\hline Esophageal Diverticulum $\dagger$ & $6(8.3)$ \\
\hline
\end{tabular}




\section{Advanced Research in Gastroenterology \& Hepatology}

\begin{tabular}{|c|c|}
\hline Gastroparesis & $5(6.9)$ \\
\hline Eosinophilic Esophagitis/Trachealization/Furrowing $\dagger$ & $4(5.6)$ \\
\hline Esophageal Ulcer† & $1(1.4)$ \\
\hline Gastric Ulcer & $3(4.2)$ \\
\hline Dilated Esophagus & $1(1.4)$ \\
\hline Esophageal Nodule $\dagger$ & $2(2.8)$ \\
\hline Esophageal Edema & $1(1.4)$ \\
\hline Varices $\dagger$ & $1(1.4)$ \\
\hline Gastro-Gastro Fistula & $1(1.4)$ \\
\hline Gastric Pouch after Bariatric Surgery & $1(1.4)$ \\
\hline Inlet Patches & $1(1.4)$ \\
\hline
\end{tabular}

†Finding considered a cause of Anatomic EGJOO

¥Several patients had multiple findings on Esophagogastroduodenoscopy

Table 4: Anatomic EGD Causes of EGJOO Identified.

\begin{tabular}{|c|c|c|c|}
\hline & Opioid Use & No Opioid Use & 39 \\
\hline Anatomic Subgroup, N & 33 & $24(61.5)$ & $43(59.7)$ \\
\hline Hiatal Hernia, N (\%) & $19(57.6)$ & $11(28.2)$ & $23(31.9)$ \\
\hline Erosive Esophagitis & $12(36.4)$ & $10(25.6)$ & $19(26.4)$ \\
\hline Stricture/Schatzki's Ring/Web & $9(27.3)$ & $3(20.5)$ & $19(26.4)$ \\
\hline Fundoplication & $11(33.3)$ & $3(7.7)$ & $6(8.3)$ \\
\hline Esophageal Diverticulum & $3(9.1)$ & $1(2.6)$ & $4(5.6)$ \\
\hline Eosinophilic Esophagitis/Trachealization/Furrowing & $1(3)$ & $1(2.6)$ & $1(1.4)$ \\
\hline Esophageal Ulcer & $0(0)$ & $0(0)$ & $1(2.8)$ \\
\hline Esophageal Nodule & $1(3)$ & $1.4)$ \\
\hline
\end{tabular}

Table 5: Treatment Results in Anatomic vs. Idiopathic Subtypes.

\begin{tabular}{|c|c|c|c|c|}
\hline & & Anatomic & Idiopathic & p-value \\
\hline \multirow[t]{7}{*}{$\begin{array}{c}\text { Treatment, } \mathrm{N} \text { (\% subcategory pa- } \\
\text { tients) }\end{array}$} & & $59(81.9)$ & $33(78.6)$ & \\
\hline & Bougie Dilation, N (\% treated) & $52(88.1)$ & $26(78.8)$ & 0.14 \\
\hline & Through the Scope Dilation (TTS) & $5(8.5)$ & $2(6.1)$ & $1.00^{* *}$ \\
\hline & Medications & $4(6.8)$ & $3(9.1)$ & $0.70^{* *}$ \\
\hline & Surgery & $4(6.8) \dagger$ & $1(3) \neq$ & $0.65^{* *}$ \\
\hline & Botox & $1(1.7)$ & $4(12.1)$ & $0.054^{* *}$ \\
\hline & Pneumatic Dilation & $0(0)$ & $9(27.3)$ & $<0.001$ \\
\hline \multicolumn{5}{|l|}{ Improvement After Treatment } \\
\hline & Yes, $\mathrm{N}$ (\% treated) & $38(64.4)$ & $21(63.6)$ & \multirow{4}{*}{$0.61^{*}$} \\
\hline & No & $9(15.3)$ & $5(15.2)$ & \\
\hline & Unknown & $12(20.3)$ & $6(18.2)$ & \\
\hline & Worsened & $0(0)$ & $1(3)$ & \\
\hline $\begin{array}{l}\text { Number of Treatment Modalities } \\
\text { before Symptomatic Improvement }\end{array}$ & & & & \\
\hline
\end{tabular}




\section{Advanced Research in Gastroenterology \& Hepatology}

\begin{tabular}{|c|c|c|c|}
\hline & One, N (\% treated with improvement) & $33(86.8)$ & $13(61.9)$ \\
\hline & Two & $5(13.2)$ & $7(33.3)$ \\
\hline $\begin{array}{c}\text { Last Treatment Modality before } \\
\text { Symptom Improvement }\end{array}$ & Three & $0(0)$ & $1(4.8)$ \\
\hline & Bougie Dilation, N (\% treated with improve- \\
& ment) & $31(81.6)$ & $9(42.9)$ \\
\hline & Surgery & $4(10.5)$ & $1(4.8)$ \\
\hline & TTS & $1(2.6)$ & $1(4.8)$ \\
\hline & TTS and Bougie Dilation & $1(2.6)$ & $0(0)$ \\
\hline & TTS and diltiazem & $1(2.6)$ & $0.047^{* *}$ \\
\hline & Pneumatic Dilation & $0(0)$ & $6(28.6)$ \\
\hline
\end{tabular}

*At least one expected cell count $<5$, Chi-square test should be interpreted with caution.

${ }^{* *}$ Due to low expected cell count, p-value from Fisher's exact test for square 2-way tables.

†Fundoplication revision x 2; Hiatal Hernia Repair x 2

‡Heller Myotomy

Hiatal hernia, esophagitis, strictures/webs/Schatzki's rings and fundoplication were seen in greater than $20 \%$ of patients with anatomic EGJOO as identified by on EGD. Gastritis was also common, however was not considered an etiology of anatomic EGJO0. Of the 43 hiatal hernias identified on EGD (note 5 patients had hiatal hernia on EGD but were deemed idiopathic secondary to the presence of abnormal emptying of TBE) only 12 (27.9\%) were identified by HRIM. Of the 26 hiatal hernias identified on HRIM, only 12 were confirmable on EGD (46.2\%). The majority of the 92 treated patients reported improvement (59 of 92, 64.1\%). By subcategory: 59 (81.9\%) patients with an anatomic etiology were treated with $38 / 59(64.4 \%)$ patients reporting symptomatic improvement, while 33 (78.6\%) patients with idiopathic etiology were treated with $21 / 33(63.6 \%)$ patients reporting symptomatic improvement, as determined by patients report of symptom resolution, reduction in severity and/or frequency. Most of the patients reporting improvement had improvement after one (33/38 86.8\% Anatomic; 13/21 61.9\% Idiopathic) or two treatment modalities (5/38 13.2\% Anatomic; 7/21 33.3\% Idiopathic), as can be seen in Table 5. One patient, who reported improvement after treatment, in the idiopathic subgroup had symptom improvement after three treatment modalities. Large bougie dilation was the treatment modality last used in patients reporting improvement with $31 / 38(81.6 \%)$ in the anatomic subgroup and $9 / 21$ (42.9\%) in the idiopathic subgroup.

\section{Discussion}

a) In our study of 114 patients the key findings were:

b) Anatomic EGJOO was more prevalent in our study than previously described in the literature $(63.2 \%$ of cases) with hiatal hernia, esophagitis, strictures/webs/Schatzki's rings and fundoplication representing the most common anatomic etiologies $[1,2,5,6,8]$.

c) Post-Surgical EGJOO is a significant but not previously well-described etiology of EGJOO seen more commonly than previously reported in our cohort.

d) Symptoms from an anatomic etiology usually resolved with treating the identified anatomic obstruction. (i.e. treating an esophageal stricture with dilation).

e) Opioid use was common at the time of HRIM in both groups, anatomic $45.8 \%$ and idiopathic $28.6 \%$.

f) In accordance with the literature, we were unable to find HRIM or radiographic parameters that differentiate the two subtypes independent of EGD. We showed that clinical presentation, liquid bolus transit, SBE, and TBE do not distinguish between idiopathic and anatomic etiologies of EGJOO. A large proportion of our study population used opioids with a greater percentage, though not statistically significant $p=0.069$, of anatomic $(45.8 \%)$ versus idiopathic (28.6\%) patients with documented opioid use. Further analysis of the anatomic subgroup shows similar incidences of the anatomic causes among opioid users and non-opioid users. EGJOO has been observed in patients with chronic opioid use raising the question if the anatomic cause, opioid use, or some combination of the two lead to the HRIM pattern of EGJOO. We do not feel opioid use was the underlying etiology of EGJOO, specifically for the anatomic group, given our treatment results as outlined below. However further evaluation into opioid use as a cofounder or the effect of opioid discontinuation on HRIM patterns and symptomatology are possible areas for future study.

Our study found that surgically altered anatomy can result in HRIM findings consistent with the manometric diagnosis of 
EGJOO and can result in clinically relevant esophageal outflow obstruction and poor esophageal emptying. The current Chicago Classification scheme is intended for patients without prior surgery however our data show that surgical changes commonly result in HRIM findings consistent with EGJOO and presenting symptoms similar to non-surgical patients. This post-surgical EGJO0, likely secondary to anatomic changes (e.g. following fundoplication), represents a significant portion of our studied total EGJOO population (21.9\%) and of our anatomic subgroup (34.7\%). This highlights a previously unaddressed cause of EGJOO patterns on HRIM which must be considered in the context of clinical history and EGD evaluation for post-surgical changes such as tight fundoplication. It also identifies an area for further expansion of the current classification scheme.

The anatomic subtype was more commonly encountered in our study (Anatomic $n=72,63.2 \%$ vs Idiopathic $n=42,36.8 \%$ ) than other studies in the current literature (which shows inversion of our results with more idiopathic cases than anatomic cases) $[1,6,8]$. Many of the identified anatomic cases have established treatments including hiatal hernias, esophagitis, strictures, and tight fundoplication. A large hiatal hernia may cause esophageal obstruction and small hiatal hernias $<3 \mathrm{~cm}$ have also been associated with the manometric diagnosis of EGJOO. This may be related to the IRP being artificially elevated by the presence of a hiatal hernia. The presence of a double pressure zone (LES and crural diaphragm) in the IRP calculation box can cause an elevated IRP when no esophageal obstruction exists. This is important as hiatal hernias are routinely encountered in clinical practice. This outlines a need to refine the computer algorithm used to calculate the IRP to prevent over diagnosis of EGJO0. It also reiterates the need for EGD to assess for a common cause of EGJOO reported on HRIM. In addition, HRIM is not able to identify prior surgical changes, esophagitis, or strictures, which are other common causes of anatomic EGJOO. As discussed above SBE and TBE were also not able to differentiate subgroups. This reiterates the need for EGD, as it is the best initial diagnostic test for differentiating between anatomic and idiopathic EGJOO.

In terms of symptomatology, dysphagia and/or typical GERD symptoms were very common among individuals with EGJOO. However, around $20 \%$ of both subgroups had no dysphagia with high proportions having a chief complaint other than dysphagia (anatomic 58.3\%, idiopathic 45.2\%). Most treated patients reported improvement of these and other presenting symptoms. There were similar rates of treatment response between the two subgroups (approximately 64\% of treated patients). However, given that the anatomic subgroup had a higher proportion of treated patients improving with just one treatment modality (86.8\% vs. $61.9 \%$ ) and a higher proportion treated with large bougie dilation ( $81.6 \%$ vs. $42.9 \%$ ), we conclude that treatment in the anatomic subgroup depends on the underlying anatomic abnormality. For example: esophageal stricture is best treated with dilation.
It is known that idiopathic EGJOO can progress to achalasia $[1,13,14]$. It is interesting that anatomic obstruction at the GE junction (pseudoachalasia) can lead to a clinical entity similar to achalasia. This is in contrast to literature discussing the pathogenesis of achalasia. Achalasia is thought to arise from ganglonitis and loss of neural inhibition of the LES but this is not the case in terms of anatomic esophageal obstruction and resultant delayed esophageal emptying that occurs at the level of the GE junction. GE junction obstruction may lead to progressive esophageal dilation to accommodate stagnate retained esophageal contents which may ultimately lead to the development of aperistalsis. This is a plausible etiology for pseudoachalasia from tight fundoplications and neoplasms at the GE junction [13].

Our study has limitations. It was a non-randomized retrospective study performed at a tertiary referral centers and may not represent clinical practice. Given the retrospective nature, clinical improvement of presenting symptoms was defined as subjective improvement documented in the clinical record and there was no standardized questionnaire administrated before and after treatment to assess patient satisfaction. Therefore, patient improvement could be influenced by recall bias. A prospective study could use objective scoring systems to define clinical improvement. We identified anatomic findings in patients with EGJOO per HRIM however ambiguity does exist on what level or severity of anatomic findings can definitively cause EGJO0. It is possible that some patients in our anatomic subgroup have incidental anatomic findings rather than an underlying anatomic etiology. Regardless identification of and consideration of anatomic findings with EGD is paramount in the evaluation of EGJ00. Study into degree or severity of specific anatomic findings needed to cause EGJOO is needed and should be prioritized by the incidence of findings we report.

Additionally, the EndoFlip ${ }^{\circledR}$ is a new high-resolution impedance planimetry device used at the time of endoscopy to measure the relationship of luminal dimensions and distensibility during controlled, volumetric distension of a high compliant balloon. Distensibility is summarized as the EGJ Distensibility Index (EGJ-DI) = narrowest cross-sectional area/intraballoon pressure for each volume. New normative values are available for Endoflip $₫$ assessment of the LES. Endoflip ${ }^{\circledR}$ has great potential as adjunct diagnostic device in the evaluation of idiopathic EGJ00. Examining the anatomic etiologies associated with the manometric diagnosis of EGJOO with Endoflip $\AA$ would be an area of future research but was not used in this study $[15,16]$. Additional provocative maneuvers such as rapid drink challenges can be used to help distinguish clinical relevance of EGJOO on HRIM however is not routinely performed at our institution. Albeit rare, malignancy has been reported in patients with the manometric diagnosis of EGJ00. Therefore, evaluation with CT imaging and/or endoscopic ultrasound (EUS) may be useful in EGJOO patients with significant weight loss to evaluate for an infiltrating process not identified on initial EGD. EUS and CT are 
costly and have associated risks and are not routinely performed at the study institution unless indicated or malignancy is suspected. Additionally, recent literature has shown no additional diagnostic benefit of cross sectional imaging and EUS $[17,18]$. No malignancy was identified in our cohort. Therefore, we do not have EUS or CT data.

Despite these limitations, our study is one of the largest studies to date examining the anatomic etiologies associated with the manometric diagnosis of EGJOO and we add new and important information to literature. Our study describes anatomic findings associated with the manometric diagnosis of EGJOO and shows these anatomic etiologies are more common than previously identified. We found altered surgical anatomy especially fundoplication is commonly associated with manometric findings consistent with EGJOO (Post-Surgical EGJ00) but is not currently considered in classification schemes. High treatment response, especially when directed to underlying anatomic causes, was identified. Opioid use was common in patients with EGJOO, the anatomic group had higher incidence of patients using opioids however not reaching statistical significance. Finally, our study confirms that EGD the best initial test to differentiate between anatomic and idiopathic EGJO0. Therefore, performing an EGD to exclude potential anatomic causes of esophageal obstruction before idiopathic EGJOO is diagnosed and treated remains paramount.

\section{Main Points}

i. Anatomic EGJOO is prevalent with hiatal hernia, esophagitis, strictures/webs/Schatzki's rings and fundoplication representing the most common anatomic etiologies.

ii. Post-Surgical EGJOO is a significant but not previously well-described etiology of EGJOO seen more commonly than previously reported in our cohort.

iii. Symptoms from an anatomic etiology usually resolved with treating the identified anatomic obstruction. (i.e. treating an esophageal stricture with dilation).

iv. Opioid use at the time of HRIM is common and was seen in anatomic $45.8 \%$ of anatomic cases and $28.6 \%$ of idiopathic cases.

\section{Author Contributions:}

Brain Jones M.D contributed to patient identification, data collection, interpretation of the data, and primary author of the manuscript. contributed to statistical analysis of the data

Steven Clayton M.D contributed to study design, patient identification, interpretation of the data, revision of the manuscript, and senior author of the investigation.

\section{References}

1. Clayton SB, Patel R, Richter JE (2016) Functional and Anatomic Esophagogastic Junction Outflow Obstruction: Manometry, Timed Barium Esophagram Findings, and Treatment Outcomes. Clin Gastroenterol Hepatol 14(6): 907-911.

2. DeLay K, Austin GL, Menard-Katcher P (2016) Anatomic abnormalities are common potential explanations of manometric esophagogastric junction outflow obstruction. Neurogastroenterol Motil 28(8): 11661171.

3. Jain A, Baker JR, Rubenstein JH, Chen JW (2017) Bolus clearance in esophagogastric junction outflow obstruction is associated with strength of peristalsis. Neurogastroenterol Motil 29(9).

4. Lynch KL, Yang YX, Metz DC, Falk GW (2017) Clinical presentation and disease course of patients with esophagogastric junction outflow obstruction. Diseases of the esophagus. Dis Esophagus 30(6): 1-6.

5. Okeke FC, Raja S, Lynch KL, Dhalla S, Nandwani M, et al. (2017) What is the clinical significance of esophagogastric junction outflow obstruction? evaluation of 60 patients at a tertiary referral center. Neurogastroenterol Motil 29(6).

6. Perez-Fernandez MT, Santander C, Marinero A, Burgos-Santamaria D, Chavarria-Herbozo C (2016) Characterization and follow-up of esophagogastric junction outflow obstruction detected by high resolution manometry. Neurogastroenterol Motily 28(1): 116-126.

7. Scherer JR, Kwiatek MA, Soper NJ, Pandolfino JE, Kahrilas PJ (2009) Functional Esophagogastric Junction Obstruction with Intact Peristalsis: A Heterogeneous Syndrome Sometimes Akin to Achalasia. Journal of Gastrointestinal Surgery 13(12): 2219.

8. van Hoeij FB, Smout AJ, Bredenoord AJ (2015) Characterization of idiopathic esophagogastric junction outflow obstruction. Neurogastroenterol Motily 27(9): 1310-1316.

9. Surgeons SoAGaE (2010) Guidelines for Surgical Treatment of Gastroesophageal Reflux Disease (GERD).

10. Kahrilas PJ, Bredenoord AJ, Fox M, Gyawali CP, Roman S, et al. (2015) The Chicago Classification of esophageal motility disorders, v3.0. Neurogastroenterol Motily ;27(2):160-74.

11. Kahrilas PJ, Ghosh SK, Pandolfino JE (2008) Esophageal motility disorders in terms of pressure topography: the Chicago Classification. J clin gastroenterol 42(5): 627-635.

12. Weijenborg PW, Kessing BF, Smout AJ, Bredenoord AJ (2014) Normal values for solid-state esophageal high-resolution manometry in a European population; an overview of all current metrics Neurogastroenterol Motily 26(5): 654-659.

13. Sanagapalli S, Sweis R (2017) Achalasia: It Is Not All Black and White. Current gastroenterology reports 19(6): 27.

14. Shin IS, Min YW, Rhee PL (2016) Esophagogastric Junction Outflow Obstruction Transformed to Type II Achalasia. Neurogastroenterol Motily 22(2): 344-345.

15. Pandolfino JE, de Ruigh A, Nicodeme F, Xiao Y, Boris L, et al. (2013) Distensibility of the esophagogastric junction assessed with the functional lumen imaging probe (FLIP) in achalasia patients. Neurogastroenterol Motil 25(6): 496-501.

16. Roman S, Gyawali CP, Xiao Y, Pandolfino JE, Kahrilas PJ (2014) The Chicago classification of motility disorders: an update. Gastrointest endosc Clin N Am 24(4): 545-561. 
17. Beveridge CA, Falk GW, Ahuja NK, Yang YX, Metz DC, et al. (2019) Low Yield of Cross-Sectional Imaging in Patients With Esophagogastric Junction Outflow Obstruction. Clin Gastroenterol Hepatol 18(7): 16431644 .
18. Liu A, Woo M, Nasser Y, Gupta M, Buresi MC, Curley M, et al. (2019) Esophagogastric junction outflow obstruction on manometry: Outcomes and lack of benefit from CT and EUS. Neurogastroenterol Motil 31(12): e12.

DOI:10.19080/ARGH.2020.16.555928 